\title{
Equipos de salud y educación para atención en Zonas Rurales
}

DR. RAMON MONTERO SCHMIDT. (*)

\section{PARTE}

Creemos que los problemas básicos de salud en Chile (malos hábitos sanitarios y alimentarios, desnutrición, abandono de menores, alcoholismo, "remedierías", trastornos conductuales de la infancia y juventud, tuberculosis, enfermedades infecciosas, parasitosis, etc.), no son afrontados por mecanismos adecuados para sus fuentes de origen. Urge crearlos o adaptarlos, aprovechando los ya existentes, que indudablemente algo han hecho, pero que no se han mostrado eficaces, como deseáramos, hasta hoy. Pensamos que en los medios rurales, suburbanos, o en los llamados marginales, se generan los mayores problemas de salud que son gestados por numerosos factores: educacionales, sanitarios, económicos, ambientales y de vivienda; pero son los dos primeros los que a nuestro juicio encadenan a los otros. Sostenemos que si logramos actuar bien en esos terrenos, los demás factores podrán ser influidos muy favorablemente. Creemos también que las disciplinas médicas y paramédicas actúan de momento aisladas y muchas veces enfrentadas, creando conflictos, y que no hay una conciencia firme en las autoridades de todo tipo sobre medicina comunitaria; sostenemos asimismo que la educación básica y media no incluyen o soslayan, en sus programas métodos que permitirían capacitarlas para disminuir el problema general de la subcultura, y por ende, el de la salud.

Es así como la educación parvularia sigue en pañales. No más del $8 \%$ de la pobla-

(*) Profesor Auxiliar, Departamento de Pediatría, Area Oriente, Universidad de Chile. ción de dos a cinco años de edad, está en condiciones de recibirla y de ésta el 50\% aún la rechaza, o por incuria o ignorancia de los padres y tutores, o por no saber adaptarla a los planes de educación general. Salud y educación (y también agronomía, arquitectura y todas las facultades universitarias), deben marchar estrictamente unidas para hacer obras trascendentes en pro de la cultura y bienestar comunitarios y no seguir actuando por separado sin intercambiar ideas y proyectos.

Los costos para adecuar lo existente al sistema que aquí propondremos no son onerosos. Es absolutamente posible afrontarlos y hay experiencias que avalan lo que aquí afirmamos.

\section{Para esto proponemos:}

1. Que se establezcan en cada región del país centros sanitario-educacionales. Estos serán de dos tipos: 1) tipo $\mathrm{A}$, con la mayor infraestructura posible, funcionarán en capitales de provincias o departamentos, y serán docentes y secundariamente asistenciales : su ejemplo más notorios, el de Lo Barnechea de Las Condes; y otros, tipo B : que serán llamados postas rurales y que serán asistenciales y de medicina preventiva y secundariamente docentes a nivel elemental, con un programa específico y sencillo (tipo el de Orilla de Auquinco, Colchagua) para capacitar especialmente a las madres en el manejo de sus hijos. 
$2^{\circ}$. En los Centros sanitario-educacionales regionales tipo A, existirá un Médico General de Zona adiestrado en el sistema (o más si la comunidad puede financiarlos), una enfermera universitaria o de la Cruz Roja entrenada, una matrona, una nutrióloga, un tecnólogo médico, una asistente social, uno o dos dentistas, una ambulancia o vehículo adecuado, un laboratorio rural, un centro de planificación familiar; deberá tener capacidad para internado rural de todas las asignaturas médicas. Tanto los centros A como los B funcionarán lo más cercanos (ojalá vecinos) a los Colegios correspondientes, cuyos programas se adecuarán para que los educadores y los educandos, desde $2^{\circ}$ año básico hacia arriba (y quizás si sería mejor que ésto sucediese aún antes), conozcan los problemas de salud que viven y reciban en ellos las enseñanzas que permitan solucionarlos en cuanto se generen, y mejor aún, prevenirlos antes que se produzcan.

Nada sacamos a veces con costosos equipos no usados, o con multiplicar hospitales, ni con tener estructuras sanitarias de espera y recibir siempre el choque de los hechos consumados, especialmente de la desnutrición y otros problemas de cualquiera edad, por no tener medios adecuados para prevenirlos y segar en su inicio la causa de estos males.

3․ Por ello creemos indispensable aumentar y destacar profesionales de salud a nivel medio rural.

Los centros sanitario-educacionales tipo $B$ no precisan de médico permanente, ni siquiera de enfermera universitaria para desarrollar una labor útil y trascendente de tipo asistencial. Dichos profesionales, entrenados especialmente en medicina comunitaria, actuarán en los Centros A, pero deberán sí, visitar y controlar en plazos determinados, las acciones de salud efectuadas en los Centros B, harán docencia y monitoría en ellos, en plazos determinados, y afrontarán la patología escogida. Si hay comunidad organizada, hecho indispensable, ella misma destacará un equipo básico de salud a nivel medio, que resuelva emergencias y que posea un número de destrezas que afronten especialmente la enfermería elemental y los primeros auxilios, que prevenga la desnutri- ción y sepa manejar los trastornos nutritivos de la infancia. Incluso dicho equipo deberá orientar en planificación familiar y preparar la acción de los profesionales de los Centros A, y de los superiores universitarios.

Estas simples acciones en rural disminuirán indiscutiblemente la presión asistencial sobre los médicos y los hospitales base departamentales, provinciales o regionales, donde llegan enfermos agravados por el tiempo y la distancia, y que han perdido por ello horas o días preciosos en viajes y esperas y desde donde muchas veces son rechazados por sobrecarga asistencial.

4\%. La profesional más idónea a nuestro juicio para los Centros B o postas rurales es la matrona, la cual recibirá un adiestramiento mayor en enfermería y cirugía menor y sobre todo en puericultura, disciplinas todas que, por lo demás, figuran en su propio curriculum.

Si no fuere posible obtener esta profesional, o la comunidad no pudiese pagarla, servirán significativamente las auxiliares de enfermeria de la propia zona, rentadas por el Estado y/o por las comunidades respectivas. Por supuesto que en este último caso es más perentorio que con la matrona el que estas profesionales sean visitadas $\mathrm{y}$ orientadas por el médico y el equipo responsable del sistema. Por experiencia creemos firmemente que debe promoverse la formación de la Cruz Roja Zonal con sus autoridades propias en todo lugar que sea posible. Esta benemérita institución presta servicios utilísimos: asistencia social, puericultoras auxiliares, personal de terreno, enfermería elemental, etc.; puede ser piedra angular del equipo de salud, y lo ha demostrado.

5․ Los educadores y educandos también recibirán entrenamiento sanitario, y actuarán solidariamente. El equipo rural sanitario-educacional comunitario, puro o comunitario-estatal, deberá quedar capacitado para vacunar, enseñar hidratación oral, higiene básica, dietética elemental, primeros auxilios, inyecciones, lavado gástrico; detectarán a tiempo la desnutrición, el retraso escolar, la sordera, las deficiencias visuales, las adenopatías, la parasitosis, la ortopedia elemental, la tuberculosis; sabrán to- 
mar muestras de sangre y orina, citar para médico los casos crónicos o difíciles; sabrán trasladar heridos, tomar la presión arterial, actuar ante quemados, ante epistaxis, ante problemas obstétricos elementales (en especial en el caso de la auxiliar de enfermería). Podrán dirigir de acuerdo con los educadores y educandos un jardín infantil dondequiera haya un colegio; orientar sobre ecología y daños a la naturaleza, sobre juegos y distracciones; informar sobre aspectos positivos y negativos del deporte, la radio y la televisión, etc.

6․ El financiamiento será regional, comunitario y al comienzo también estatal. Nada se hará gratis, las comunidades deberán estar conscientes que deben ellas mismas preocuparse del desarrollo y educación. En las regiones donde la subcultura no tenga las iniciativas necesarias, ayudarán organizaciones pertinentes: universidades, especialmente Medicina, Economía y Arquitectura, Servicio Social de la Juventud, CEMA, Voluntariado Nacional y las Fuerzas Armadas, que al comienzo de este plan serán decisivas para su puesta en marcha.

\section{En resumen :}

Creemos que un equipo de salud y educación ideal para rural es de momento difícil de conseguir por su alto costo y déficit de profesionales, sean éstos médicos, enfermeras, matronas, nutriólogas, odontólogos, parvularias, asistentes sociales, etc.

Pero podemos y debemos, dada la urgencia de los problemas de salud, destacar en rural (o localidades suburbanas pequeñas o marginales), un equipo zonal de preparación media con entrenamiento adecuado y control responsable que afrontará los problemas de salud, especialmente preventivos, en forma trascendente y útil.

El profesional más adecuado en estas acciones es la matrona, o a falta de ella las auxiliares de enfermería zonales, ya sean estatales o pertenecientes a la Cruz Roja Local; profesionales todas que serán estipendiadas por sus servicios, sin perjuicio de la colaboración absolutamente indispensable que recibirán de los educadores y educandos, de los voluntarios y de las instituciones o personas que la comunidad organizada destaque.

\section{PARTE}

\section{PRINCIPALES ACCIONES A REALIZAR A NIVEL RURAL POR LOS EQUIPOS DE SALUD Y EDUCACION COMUNITARIOS.}

\section{Atención de morbilidad}

Correcta scmiología y prescripción. Evitar "remedierías" y yatrogenias (Tetraciclinas en niños pequeños, abusos con Vitamina D y Aspirinas), se aprovechará para enseñar higiene y eliminar malos hábitos ("tónicos", abrigos excesivos, chupetes, etc.).

\section{2\%. Diagnosticar en profundidad}

No enfatizar resfríos, no dejarse abrumar por patología intrascendente. Consultar al monitor o docente. Observar con detención si hay desnutrición, infección urinaria, retardo pedagógico o mental, estreptococia crónica, sinusitis, otitis agudas o crónicas ; pesquisar la tuberculosis (PPD), malformaciones dentarias y caries, criptorquidias, parasitosis, hipocromías. Se pesquisará la enfermedad reumática, los trastornos de conducta, los déficit de escolaridad, la constipación, las diarreas crónicas. Se investigará la agudeza visual y auditiva, la dislexia, la displasia de caderas, y en general la ortopedia elemental; los malos hábitos de nutrición (golosinas, pan excesivo, horarios caprichosos, bebidas gaseosas a todas horas, chicles, cigarros, en los escolares, uso de drogas ).

\section{3?. Enseñanza y preparación de Madres}

Verificación constante del aprovechamiento que obtienen principalmente en dietética e higiene. Clasificar a las madres según $s u$ responsabilidad, capacidad de aprender y de mantenerse en lo enseñado, sin revertir a los hábitos erróneos. Niño sin madre capacitada es candidato a enfermarse, desnutrición, escolaridad deficiente, sobreprotección, problemas de conducta, posiciones antisociales o negativas. Por ello se insistirá una y otra vez en la lucha contra los malos hábitos y la tendencia inveterada a mantenerlos. Se enseñará repetidamente el lavado de manos, el transporte y el vestuario adecuados del niño pequeño.

4․ Visitar hogares con equipos de salud y educación, de donde provengan desnutridos, parasitados o retardados; enseñar 
cuidado de animales domésticos y sus enfermedades más frecuentes y trasmisibles, limitación de perros, vacunación de éstos. Indicaciones sobre ventilación y luz, sobre aglomeraciones, manejo del agua y eliminación de excretas, uso del jabón, saber bañarse; pesquisa del alcoholismo. Comprobar aprovechamiento escolar con los maestros y alumnos y alfabetización por éstos a domicilio de los adultos renuentes, especialmente madres. Vigilar a los párvulos, propensos a accidentes y a los malos ejemplos (edad de la comprensión inicial ). Formar Jardines Infantiles que puedan ser atendidos por las Unidades Educacionales Regionales con supervisión técnica. Orientar ante los fenómenos que desencadenan la radio y la TV. Influencias positivas y negativas.

\section{5․ Preparación de personal paramédico $y$ auxiliar de la propia comunidad}

Asistencia de la Cruz Roja local. Instrucción sanitaria de maestros y alumnos de los últimos cursos, llevarlos por grupos al Centro de Salud; que vean y aprendan sobre quemaduras, transportes de heridos, desnutridos, malos hábitos y de cómo se afrontan estos hechos. Saber dar charlas en las Escuelas y Colegios sobre temas previamente analizados con los docentes y monitores y de acuerdo con los maestros, quienes solicitarán temas a desarrollar. Interesarse y participar con la juventud de la zona sobre todo en lo referente a Medicina del Deporte y en los peligros del alcohol, al tabaco y las drogas. Interesarse mucho por la ecología (protección de la naturaleza y al ambiente) y difundirla con los maestros y educadores de todo orden. Conocer la flora y fauna silvestre de la zona. Informar sobre contaminación, abuso de herbicidas y pesticidas (especialmente DDT) peligros de la destrucción del bosque, de las aves silvestres y de sus nidos; lucha contra las ratas. Enseñar las vedas. Eliminación de las hondas y de la pesca delictiva.

\section{6․ Aprender y saber enseñar nutrición básica.}

Destruir el mito del "ulpo", gran agente de la desnutrición; del "desayuno del pobre", o sea, el té con azúcar y el pan excesivo. Del miedo y rechazo de los alimentos crudos. Mostrar el verdadero valor (dietético y económico) de los quesos, las carnes, peces, hortalizas, miel, del conejo, del repollo, de las sardinas, del pescado seco, de las hortalizas de invierno (que por ignorancia casi no las cultiva nadie en la zona); del manejo de la culinaria elemental con gasto de combustible excesivo y peligro de quemaduras para los pequeños; de la higiene permanente en la manipulación de alimentos, de no guardar pesticidas junto a ellos, de no dar de comer a los niños a deshora, del uso de la olla a presión, de las ventajas del aceite sobre las mantecas, del uso correcto de los huevos, de la manera de conservar alimentos y del abuso del pan. Uso de los alimentos proporcionados por el SNS y por otras instituciones. Todo esto irá coordinado con los líderes comunitarios y con los maestros y alumnos.

$7^{\circ}$. Aprender para después enseñar: primeros auxilios, cirugía menor y enfermería básica en el mismo Centro de Salud. Verificar lo enseñado, aprender y enseñar lavado gástrico, enemas, aspiración de secreciones, extracción de sangre y fleboclisis, criterio ante quemaduras, ante vómitos incoercibles, hipertemia, convulsiones, alergia aguda, intoxicaciones frecuentes, suturas, hemorragias, traumatismo, ortopedia elemental, transporte de heridos, obstetricia elemental, en general, poner las destrezas indispensables para actuar y enseñar en zona rural.

8. Planificación familiar: desarrollar un programa adecuado de protección a la familia; paternidad responsable. Coordinar con los maestros de las escuelas y con los docentes y monitores universitarios y de la zona. Educación sexual, discutida con ellos y apropiada a la mentalidad de los habitantes para evitar exageraciones.

9․ Aprender y poder enseñar el uso correcto y el cuidado de los Centros SanitarioEducacionales Rurales y saber el aseo de los locales, aspiración de polvo, funcionamiento de ventiladores y estufas, cuidado de la ambulancia y equipo. Observación crítica de lo notado durante su asistencia al Hospital $\mathrm{Ba}$ se y locales escolares. Dar y aceptar insinuaciones, analizar estos hechos con docentes y monitores. Saber llevar relaciones de convivencia con las autoridades civiles - militares - religiosas, etc., y sobre todo con la comunidad. Saber orientar respecto a radio, TV, deportes.

\section{0?. Como objetivo fundamental quedar ca-} pacitados para poder ser ellos monitores o premonitores del Sistema, al ser éste extendido a todo el País, cuando empiece a funcionar el Servicio Social de la Juventud, herramienta absolutamente indispensable junto al Servicio Social Universitario pa- 
ra que este plan Sanitario-Educacional rinda sus frutos y ayude a erradicar la subcultura y sus consecuencias : la desnutrición y la pobreza intelectual.

Por ello proponemos poner en marcha cuanto antes el siguiente Proyecto de Internado Rural para Pre-graduados de Medicina, Obstetricia, Odontología y Enfermería, basado en los puntos siguientes:

19. Destacar en Rural o en regiones suburbanas grupos de alumnos del último curso de dichas carreras o pre-graduados con monitorías adecuadas en los Centros Sanitario-educacionales existentes ( $\mathrm{A}$ o $B$ - véase anteriormente), y hacer que cumplan con los equipos de los Centros A allí existentes un plan de enseñanza para protección de la salud. Ya entrenados actuarán después en los Centros B.

Las Comunidades les proporcionarán los locales adecuados y los medios de subsistencia y el Estado, las FF.AA. y nuevamente las comunidades, los medios de movilización.

2․ Se comenzará por Regiones hasta extodo el país. tender el Sistema gradualmente a

3․ Las Universidades (especialmente la Facultad de Medicina, Agronomía, Arquitectura), el Estado y las Organizaciones Comunitarias, en acción conjunta con equipos directivos experimentados y pequeños, iniciarán en regiones, diversos planes pilotos con estas ideas y evaluarán resultados.

El Sistema Lo Barnechea (Santiago), Auquinco, Chépica, La Orilla (Colchagua), puede servir desde ya como ejemplo del Sistema.

\section{PARTE}

\section{Destrezas que debe adquirir y saber enseñar el Interno o Pre-graduado en su estadia en Rural (Internado)}

A) ¿Cuál es dentro de una encuesta sencilla el estado socio-sanitario de una comunidad? ¿Qué medios elementales se requieren para conservarlo en forma y para que no se generen enfermedades en él? Ejemplos: Manejo del agua y alimentos, escolaridad, alcoholismo, ingresos económicos.
B) Conocer los hábitos existentes, deshacer los peligrosos y crear o activar los valederos:

19. En alimentación: Hay poco y mal uso de leche y derivados, temor a priori a los alimentos crudos y manipulación anti-higiénica de ellos. Poco consumo de verduras y de fuentes de proteínas que no sean carne de vacuno o de ave, mal empleo del huevo (generalmente lo venden) y menosprecio parcial o total de muchos peces, de los conejos, cuyes, etc. Exaltación del pan, de las frutas cocidas, y de las bebidas gaseosas, del llamado ulpo, y en general de las harinas. Malgasto de combustible. Desaprovechamiento del agua de cocción de verduras y leguminosas, peladura de las papas y uso exagerado de purés. Desaprovechamiento de frutas de la estación; no hay conocimientos sobre conservación de alimentos; consumo habitual de frutas verdes o "pintonas". No hacen huerta de invierno. Desnaturalización de las carnes (cocimiento excesivo); desaprovechamiento de vísceras y sangre animales. (en numerosos mataderos la botan, ej.: Nancagua y varias regiones de Colchagua). Poco consumo de quesos, usan leches diluidas, hay ignorancia sobre manejo y prescripción de leches en polvo, poco uso de sardinas y pescados desecados, exceso de té y azúcar.

29. En higiene elemental: Indiferencia ante la suciedad ambiente (revisar las manos infantiles); las habitaciones no las pintan ni aspiran, son grises, de ventanas pequeñas, casi sin luz ni ventilación; norias y pozos negros frecuentemente vecinos; animales de todo tipo deambulan sueltos por la casa, y aún por las habitaciones, perros $\mathrm{y}$ gatos excesivos, jabón casi no se usa; sobre moscas no hay conciencia de matarlas, sólo las espantan; lo mismo sucede con pulgas y chinches, no saben combatirlas; miedo ancestral al agua y al aire, higiene dental inexistente "emboticamientos" y "remedierías" son doctrinas de alto valor; se produce desilución cuando los médicos no prescriben muchos "remedios"; prestigio de tónicos, jarabes para la tos y medicinas diversas, recetadas por cualquiera.

3' Urge, entonces, enseñar a la madre el manejo correcto de las diversas enfermedades de la infancia con enfatización de los puntos 1 y 2 de esta exposición; además que sepan bañar y vestir a sus hijos (no abrumarlos con lanas, chales y gorros), insistir en los daños del chupete y de la 
transpiración, en los inconvenientes de la sobreprotección o de los castigos y golpes, que adquieran conciencia de que el control del niño sano es imprescindible, y que debe ser hecho cada dos meses en el primer año de la vida, y cada tres, en el segundo año. Pensamos que no debe exigirse estos controles cada 30 días como hasta hoy. Son excesivos y fatigan a las madres. Deben mantenerse sólo en familias con alto riesgo social y para ello insistimos una vez que es indispensable clasificarlas según sus conocimientos y responsabilidad.

4. Tener nociones de uso adecuado de juguetes, de educación parvularia, y estar capacitados para ayudar en esta disciplina, absolutamente necesaria en comunidades urbanas pequeñas y medianas, en que hay más aislamiento cultural. Insistir en conceptos sobre paternidad responsable (no olvidar que el padre chileno, especialmente el subculto, tiene en general mala conducta social).

5. Saber vacunar y enseñar esta destreza con fundamentos teóricos.

6. Saber enseñar primeros auxilios, difundir pautas de este tipo en las enfermeras universitarias y de la Cruz Roja; enseñar así criterios ante traumatizados de cráneo, quemados, intoxicados y parasitados, o ante epidemias o endemias locales: sarna, impétigo, estreptococias en general, salmonellosis. Transporte y traslado de enfermos, higiene dental.

$7^{\circ}$ Tener ideas claras e inculcarlas sobre lactancia materna, insubstituible en los primeros meses; sobre la pesquisa de niños limitados, con convulsiones, sordos, disléxicos, enuréticos, con daños psiconeurológicos o alteraciones de conducta; sobre tuberculosis, enfermedad reumática, alcoholismo, infección urinaria y sobre todo sobre desnutrición y trastornos nutritivos agudos; su consulta oportuna y tratamiento adecuado.

8. Saber preparar personal paramédico y lideres comunitarios que sean de la localidad, para que aprendan las destrezas señaladas. Saber controlar y evaluar permanentemente el aprendizaje de estos valiosos elementos humanos.

99. Saber convivir con los habitantes; promover a la juventud al deporte y a la protección de la naturaleza; alternar con las autoridades locales y con las creencias religiosas de todo tipo.

10' Poder manejar un laboratorio rural que efectúe un mínimo de 5 exámenes sencillos y corrientes : sedimentación globular, hematocrito, microscopía de heces, examen de frotis faríngeo de secreciones, sedimento urinario.

Tiene por último que quedar capacitado el pre-graduado para afrontar estos hechos de honda raigambre sanitario-cultural, y deben arbitrarse los medios para conectar a todas las Facultades Universitarias, y a toda la institucionalidad nacional en estos problemas, raíces de la insalubridad, de la extrema pobreza y de sus consecuencias.

Arquitectura, Odontologia, Veterinaria, Agronomía, etc., y por supuesto Medicina y sus proyecciones, deben iniciar de una vez por todas y en conjunto, no actuar aisladas, este camino. Lo conocemos y es por eso que lo propiciamos con verdadera majadería y pertinacia.

La Salud no depende de los médicos, ni la Medicina consiste en tratar secuelas y enfermedades, sino que fundamentalmente en adelantarse a ellas y así evitar los daños humanos y económicos que la insalubridad produce.

\section{REFERENCIAS}

Sistema integrado para la supervisión sanitaria. Janice C. Levy, Robert M. Strong, y col. Clinicas Pediátricas de Norteamérica; Mayo, 1974.

Jaque al Subdesarrollo. Fernando Monckeberg Edit. Gabriela Mistral. 1974.

Seminario Internacional de Pediatría y Bienestar de la Familia, Paraguay, Noviembre, 1973. Jorge Hamuy, Paraguay: Werner Bustamante E., Chile; Renán Esquivel, Panamá; Juan Carlos Ivetta, Argentina; Dámaso Villarroel, Venezuela; Fernando Hurtado Leverton, Colombia; J. Luis Delgadillo, Paraguay; Badi Teme Lichi, Paraguay; Gastón Moscoso Zamora, Bolivia; Ramón Montero Schmidt, Chile.

Organización del equipo asistencial primario. Benjamin Siegel - Centro de Asistencia Primaria, Departamento de Pediatría, Universidad de Boston, Mass. Clínicas Pediátricas de Norteamérica; Mayo, 1974. 
Centros sanitario-educacionales. La experiencia de Lo Barnechea. Dr. Ramón Montero Sch. y colaboradores. Congreso Nacional de Pediatría, Santiago. Mayo, 1973.

Demografía y Epidemiología. Dr. Dámaso Villarroel. Universidad Central de Venezuela. 1972. Publicación ante la Facultad de Medicina.

Salud y Hospitales. Dr. Antonio Montero Carvallo. Edit. Periodística de Chile, 1973.

Protección Familiar. Un programa de salud pública. Dr. Roberto Kriskovich. Seminario de Pediatría y Bienestar Familiar. Nov. 1973. (Paraguay).

Salud Materno-Infantil. Luis Givovic. Rev. Ch. Ped. 44: 259, 1974.

Lactancia Natural. Patricia Marin. Rev. Ch. Ped. 45: 259, 1974.

Tratamiento de la Desnutrición. Alejandro Maccio. ni. Rev. Ch. Ped. 45: 161, 1974.
Revista Chilena de Pediatría. Vol. 44 № 6: Nutrición. Dres. M. Eugenia Rodriguez, Ema Valle. jos, Eliana Fernández, J. Torres, J. Martínez, Sergio Jarpa, Fernando Monckeberg, Francois Biancani, Ana María Kaempfer, Germán S. Otto, Ives Lacassie, V. Gattas, M. Aguayo, J. Hasbún, Luis Givovic, Jorge Martner y otros.

Actitudes y Conductas de Lactancia en 100 madres de Santiago. Rev. Ch. Ped. 44: 475, 1973. Dres. Karen Neves, Marta Valenzuela, Margarita Araya, Fernando Monckeberg.

Estudios en Malnutrición Fetal. Drs. Mafalda Ri. zardini, Julio Meneghello, Gastón Duffau, J. Contreras. Pediatría. Santiago, 19-89, 1969.

Escuela para Padres. Dra. Eva Giberti, Buenos Aires, Rep. Argentina. 1972.

Un Programa Integrado de Salud y Planificación Familiar en el Distrito Estimesgut de Turquía. Nusret H. Fisek. Estudios de Planificación Familiar, Julio, 1974. 